\title{
Evaluando el logro de los principios de la gestión de la calidad en empresas constructoras certificadas según ISO 9001:2000
}

\section{Evaluating the attainment of quality management principles in construction companies certified by ISO 9001:2000}

\author{
Tania Romero*1, Alfredo Serpell* \\ * Departamento de Ingeniería y Gestión de la Construcción, Pontificia U niversidad Católica de Chile, Santiago, CH ILE \\ Fecha de recepción: 10/ 08/2007 \\ Fecha de aceptación: 15/11/2007 \\ PAG. $197-213$
}

Resumen

\begin{abstract}
Desde mediados de los años noventa, las empresas constructoras habitacionales chilenas comenzaron a incorporar la gestión de calidad y han implementado sistemas de gestión de la calidad (en adelante SGC) optando principalmente, por utilizar las directrices dadas por la familia de normas ISO 9000. LaS constructoras han invertido una gran cantidad de recursos, especialmente económicos y humanos, en implementar sus SGC con el fin de aumentar la satisfacción de sus clientes a través del mejoramiento de la calidad de sus procesos constructivos y de la calidad de las viviendas que construyen. Entonces, para ellos es muy importante saber si esta inversión está dando los frutos que esperan. Por lo anterior, se realizó una investigación para determinar el impacto real de los SGC en el desempeño de las empresas constructoras certificadas ISO 9001:2000 en cuanto al nivel de aplicación de los principios de la gestión de la calidad sin considerar el impacto que puede generar la implementación de otro tipo de normas como las de manejo ambiental y de prevención de riesgos. Para lograrlo, se empleó un instrumento de medición que consistió en una encuesta aplicada como entrevista personal, que fue respondida por 118 personas de 11 constructoras habitacionales chilenas, de un universo de 34 empresas certificadas ISO 9001:2000. Estas personas entregaron sus percepciones sobre el cumplimiento de los principios de gestión de la calidad en la empresa. El principal resultado de este estudio indica que las personas perciben que los principios de gestión de la calidad se están aplicando de manera insuficiente.
\end{abstract}

Palabras Clave: Sistema de gestión de la calidad, ISO 9001:2000, construcción, empresas constructoras, evaluación

Abstract

Since the nineties, housing construction companies began to incorporate the quality management and have developed quality management systems (Q MS), using the directives given by the ISO 9000 standards. Construction companies have invested a big amount of resources -especially economic and human resources - in the development of their Q MSs; this has been done in order to increase the level of satisfaction of their customers through the improvement of the quality of their constructive processes and the quality of the houses they build. Then, it is very important to construction companies to know if this investment has been as profitable as they hoped. A study was performed to determine the impact of Q M Ss on the performance of ISO 9001:2000 certified housing construction companies regarding the level of application of quality management's principles without considering the impact that can generate the implementation of other standards such as environmental management and risk prevention. The measuring instrument consisted of a survey applied as a personal interview, which was answered by 118 individuals from 11 Chilean housing construction companies; out of a total universe of 34 ISO $9001: 2000$ certified housing construction companies. These people gave their perceptions over the accomplishment of quality management's principles in their companies. The main conclusion of this study indicates that people perceive that quality management's principles are applied in an unsatisfactory way.

Keywords: Q uality management systems, ISO 9001:2000, construction, companies, evaluation

\section{Antecedentes}

Los SGC tienen como propósito mejorar continuamente el desarrollo de productos y procesos.
Para lograrlo, estos sistemas promueven el uso del control estadístico de la calidad, la importancia del liderazgo de la gerencia superior y la asociación cliente - proveedor (Evans y Lindsay, 2000), de acuerdo a las

\footnotetext{
1 Autor de correspondencia / Corresponding autor: Vicuña Mackenna 4860, Macul, Santiago, Chile.

Teléfono: (56) 02354 7179, E-mail: tromero@ing.puc.cl
} 
ideas difundidas por W. Edwards Deming desde la década de los 50. En los años siguientes, estas ideas se adoptaron en Estados U nidos y Europa, creándose posteriormente la serie de normas ISO 9000 .

Esta serie de normas se enfocan en 3 puntos específicos: 1) en el análisis de los requisitos del cliente, 2 ) en definir los procesos que aportan valor a los productos y 3) en mantenerlos bajo control (ISO 9000:2000).

En Chile, la instauración de los SGC recién comenzó en la década de los 90 . A diferencia de otros países que han seguido las directrices de distintas filosofías y normas, aquí se han seguido casi únicamente las dictadas por la familia de normas ISO 9000, adaptadas como $\mathrm{N}$ ormas Chilenas $\mathrm{O}$ ficiales desde 1994. Actualmente la versión vigente es la del año 2000.

En general, la instauración de un SGC puede dividirse en dos etapas: 1) Etapa de Implementación y 2) Etapa de M ejoramiento. Ambas etapas se han estudiado desde distintos puntos de vista como se describe a continuación.

En el ámbito internacional, las investigaciones sobre la etapa de implementación se han enfocado a determinar las metodologías y herramientas más utilizadas y más efectivas en la implementación de los SGC (relacionados con implementación de un SGC en general: Chan y Suazi, 2002; Vass y Kincade, 1999; Cameron y Sine, 1999; Michalisin y White, 2001 y, Salegna y Fazel, 1995; relacionados con la determinación de las herramientas más utilizadas: Jayaram et al. 1997; Roethlein et al., 2002; Granzol y Gershon, 1997; Chan y Q uazi, 2002; Yu et al., 1998; Sun, 2001; Choon y W isner, 2001; Jain y Tabak, 2002 y, Detert y Jenni, 2000). En la etapa de mejoramiento, se han enfocado principalmente a estudiar la relación entre la gestión de la calidad y la productividad (Kontorghiorghes, 2003 y, Mc Craken y Kaynak, 1996) y a determinar una metodología para medir la satisfacción del cliente (Reeves et al., 1995; Spencer y Crosby, 1997; G ustafsson et al., 2000 y, Torbica y Stroh, 2000). Todo esto orientado a la industria manufacturera principalmente, aunque también a la de servicios y a la educación.

En el ámbito nacional, las investigaciones de la etapa de implementación se han enfocado a desarrollar metodologías de diagnóstico de la gestión de la calidad antes de implementar un sistema formal (Figari, 1996 y O choa, 1998), a estudiar las barreras que impiden 0 dificultan la implementación de estos sistemas (González, 2001) y finalmente, las herramientas o técnicas que se utilizan para implementarlos (Molina, 1998).

En la literatura nacional no se han encontrado investigaciones relacionadas con la etapa de mejoramiento, es decir, no se ha llegado al punto de evaluar si los SG C están dando los resultados esperados en cuanto a beneficios monetarios, mejoramiento de la calidad de los productos y mejoramiento de la eficiencia de los procesos. No se conoce el impacto real de la filosofía de gestión de la calidad y de la certificación en las empresas ya certificadas ISO 9001.

\section{O bjetivos específicos e hipótesis}

El estudio realizado que aquí se informa consideró como principales objetivos, los siguientes:

A. Determinar si en el desempeño de las empresas constructoras de viviendas certificadas bajo la norma ISO 9001:2000, se respetan los principios de gestión de la calidad.

B. Determinar si la calidad de los procesos de construcción de viviendas ha mejorado como resultado de la implementación de un SGC.

C. Determinar si la calidad de las viviendas ha mejorado como resultado de implementar un SGC en su construcción.

De acuerdo a los objetivos expuestos anteriormente, se plantearon las siguientes hipótesis para esta investigación:

A. Las empresas que tienen un SGC certificado cumplen apropiadamente los principios de gestión de la calidad.

B. Las empresas que tienen un SGC certificado han logrado un mejoramiento de la calidad de los procesos de construcción.

C. Las empresas que tienen un SGC certificado han logrado un mejoramiento de la calidad de las viviendas.

En este artículo sólo se tratará el primer objetivo específico y su hipótesis asociada. Los otros objetivos se tratarán en artículos posteriores.

\section{Descripción general de la investigación}

La investigación realizada se basó en la aplicación de una encuesta a modo de entrevistas personales a 118 personas de 11 empresas constructoras 
chilenas de viviendas de un universo total de 34 empresas de esta categoría, certificadas ISO 9001:2000, por lo que se obtuvieron respuestas a las encuestas y además, comentarios y justificaciones de dichas respuestas.

En las siguientes secciones se presenta el marco teórico que sirvió para elaborar la encuesta, la metodología seguida para determinar el tamaño muestral, la confección de la encuesta y el análisis de los resultados obtenidos. Posteriormente, se presentan las conclusiones y las perspectivas futuras.

Cabe mencionar que no es parte del alcance de esta investigación analizar la aplicación de los Sistemas de Gestión de la Calidad en las empresas subcontratistas.

\subsection{M arco teórico}

Un SGC basado en la norma ISO 9000:2000 proporciona a las organizaciones un marco de referencia para la mejora continua y proporciona confianza en que los procesos utilizados son capaces de generar productos de las características definidas por la organización (la organización determina las características de sus productos en base al estudio de las necesidades y expectativas del cliente). Esto es así, porque un SGC se focaliza en el análisis de los requisitos del cliente, en definir los procesos que aportan a la elaboración de productos aceptables para el cliente y en mantener estos procesos bajo control a través de las etapas detalladas a continuación (ISO 9001:2000):

a. Determinar las necesidades y expectativas de los clientes y de otras partes interesadas.

b. Establecer la política y objetivos de la calidad de la organización, con el fin de tener un marco de referencia para dirigir la organización, determinar los resultados deseados y ayudar a destinar los recursos disponibles para alcanzar dichos resultados.

c. Determinar los procesos y las responsabilidades necesarias para el logro de los objetivos de la calidad.

d. Establecer los métodos para medir la eficacia y eficiencia de cada proceso.

e. Aplicar estas medidas para determinar la eficacia y eficiencia de cada proceso.

f. D eterminar los medios para prevenir no conformidades y eliminar sus causas.

g. Establecer y aplicar un proceso para la mejora continua del sistema de gestión de la calidad.

\subsubsection{Principios de gestión de la calidad}

La norma ISO 9001:2000 identifica ocho principios que pueden ser utilizados por la dirección para mejorar continuamente su desempeño. Estos principios son los siguientes y fueron utilizados como variables de la investigación:

- Enfoque al cliente

- Liderazgo

- Participación del personal

- Enfoque basado en procesos

- Enfoque de sistema para la gestión

- Mejora continua

- Enfoque basado en hechos para la toma de decisión

- Relaciones mutuamente beneficiosas con el proveedor

\subsection{M etodología de investigación}

\subsubsection{Determinación del tamaño muestral}

En primer lugar, se recolectó la información para determinar el universo de constructoras habitacionales certificadas ISO 9001:2000 (no existe un listado oficial de empresas certificadas bajo esta norma), con lo que se llegó a identificar 34 constructoras habitacionales certificadas ISO 9001:2000 en todo el país, de las cuales 27 (79\%) tienen su casa matriz en la Región Metropolitana.

Entonces, considerando el reducido tamaño del universo, se decidió realizar un censo a las constructoras con casa matriz en la Región Metropolitana. Se recibió respuesta de 11 de ellas, las que representan el 32\% del total.

Al interior de cada empresa, la muestra se compone, como mínimo, de una persona de cada nivel jerárquico de la línea de producción, desde el Gerente General hasta el nivel de capataces. Para esto, se entrevistó a personal de la oficina central y a personal de una obra de cada empresa. Se dejó a la empresa escoger la obra donde se haría la investigación con la condición era que fuera la más representativa del desempeño del SGC. El sesgo que puede resultar de esta elección se considera minimizado, porque las encuestas se contestaron a través de una entrevista, lo que permitió profundizar algunos temas, consultar por las otras obras de la empresa y detectar ciertas contradicciones. Además, se entrevistó a personas que se relacionan sólo con una obra en particular y a personas que se relacionan con todas las obras de la empresa, lo que permitió obtener las percepciones de estas personas en cuanto a las diferencias en la aplicación del Sistema de Gestión de la Calidad en las distintas obras (lo que se reflejó en las respuestas a la encuesta). 


\subsubsection{Caracterización de la muestra}

La muestra está formada por 118 personas que trabajan en 11 empresas constructoras habitacionales chilenas. Esta muestra se dividió de acuerdo a dos puntos de vista con el fin de verificar si existen diferencias en la percepción del desempeño del SG C entre los distintos grupos: primero, de acuerdo al lugar de trabajo de los encuestados (tabla 1) y segundo, de acuerdo al nivel educacional de los encuestados (tabla 2).

La mayoría de los encuestados son profesionales y también la mayoría trabaja directamente en obra.

Tabla 1. Lugar de trabajo de los encuestados

\begin{tabular}{||c|c|c||}
\hline $\begin{array}{c}\text { Lugar de } \\
\text { Trabajo }\end{array}$ & $\begin{array}{c}\mathbf{N}^{\circ} \\
\text { Encuestados }\end{array}$ & $\%$ \\
\hline Oficina Central & 34 & $29 \%$ \\
\hline Obra & 84 & $71 \%$ \\
\hline
\end{tabular}

Tabla 2. Nivel educacional de los encuestados

\begin{tabular}{|c|c|c||}
\hline $\begin{array}{c}\text { Nivel } \\
\text { Educacional }\end{array}$ & $\begin{array}{c}\mathbf{N}^{\circ} \\
\text { Encuestados }\end{array}$ & $\%$ \\
\hline Profesionales & 72 & $61 \%$ \\
\hline Técnicos & 46 & $39 \%$ \\
\hline
\end{tabular}

\subsubsection{Instrumento de medición (Encuesta)}

El instrumento de medición utilizado en esta investigación fue una encuesta, aplicada a través de una entrevista.

Se escogió la encuesta como método de obtención de datos porque, de acuerdo a lo descrito por Salkind (1999), "... indaga sobre constructos como actitudes, creencias, prejuicios, preferencias y opiniones".

Específicamente, esta investigación pretende obtener las percepciones de las personas respecto de la utilización y efectividad de los SGC, por lo tanto, este instrumento se acomoda al objetivo. Además, se prefirió aplicarla a través de una entrevista para tener la oportunidad de profundizar en los temas más importantes para la investigación y/o en los de interés del entrevistado.

Cabe mencionar que no se consideró como requisito que los entrevistados tuvieran conocimientos previos sobre Gestión de la Calidad, ya que las preguntas fueron redactadas de tal forma que no fue necesario que el entrevistado conociera y entendiera la definición de cada principio. Más bien, los encuestados debían verificar si las situaciones planteadas en la encuesta existían en su lugar de trabajo o no.
La encuesta se dividió en cuatro partes. En este artículo sólo se analizarán los resultados de la primera parte (se presenta en el Anexo A), correspondiente al primer objetivo específico. Esta sección se compone de 41 afirmaciones orientadas a determinar el grado de cumplimiento de los principios de la gestión de la calidad en los que se basa la norma ISO 9001:2000. Las afirmaciones se contestaron en base a escalas tipo Likert de siete puntos y además, se registraron las observaciones y comentarios que los encuestados hicieron a las respuestas de la encuesta.

Se realizó una prueba piloto para evaluar la comprensión de los ítems incluidos en la encuesta y corregir los errores detectados. La aplicación de la encuesta se realizó entre Enero y Julio de 2006.

La confiabilidad del instrumento de medición se calculó mediante el coeficiente Alfa de Cronbach ( $\alpha$ del instrumento $=0.973$ ) y se corroboró con la correlación ítem-total. A través de la utilización de estos dos índices se eliminaron los ítems que no eran consistentes con los resultados del instrumento.

De acuerdo a lo anterior, se eliminaron 7 preguntas que estaban dirigidas a determinar el cumplimiento de los siguientes principios de gestión de la calidad: una pregunta sobre el liderazgo, dos preguntas sobre la participación del personal y cuatro preguntas sobre la mejora continua. Para los análisis siguientes se consideró el instrumento sin las preguntas eliminadas.

Posterior al análisis de confiabilidad se realizó un análisis factorial con dos objetivos: 1) obtener puntajes estandarizados para cada encuestado y 2) determinar si cada variable apunta a medir solo el aspecto que se pretende o se subdivide en más de uno. Las ponderaciones resultantes para cada ítem se presentan en el Anexo B. Los resultados arrojaron que los principios de gestión de la calidad "enfoque en el cliente", "mejora continua" y "relaciones mutuamente beneficiosas con el proveedor" presentan dos dimensiones, el resto de las variables sólo una.

El principio de gestión de la calidad "enfoque al cliente", se refiere al cliente externo (quien comprará las viviendas). El análisis factorial determinó que esta variable, en realidad corresponde a 2 dimensiones 0 variables, que se llamaron "relación directa" y "relación indirecta". La primera mide todo lo concerniente la relación de la constructora con el comprador de la vivienda y la segunda mide lo concerniente a la relación de la constructora con el comprador, a través de la inmobiliaria.

Los resultados también arrojaron que el principio de gestión de la calidad "mejora continua" presenta dos 
dimensiones, las que se llamaron "difusión de prácticas" y "mal uso de herramientas". La primera, se refiere a la difusión de las prácticas de gestión de la calidad (herramientas para mejorar continuamente la calidad de productos y procesos) al interior de cada empresa y la segunda se refiere al mal uso de ellas.

El principio de gestión de la calidad "relaciones mutuamente beneficiosas con el proveedor" presenta dos dimensiones que fueron Ilamadas "relación contractual" y "relación extra-contractual". La primera se refiere a todo lo que queda estipulado por escrito entre la constructora y el proveedor (de materiales o servicios) y la segunda, se refiere a los acuerdos tácitos entre ambas partes.

Desde ahora en adelante estos tres principios de gestión de la calidad se considerarán como 6 variables separadas y se diferenciarán por los apellidos recién explicados.

\section{Resultados}

El análisis de resultados tratará sobre los siguientes aspectos:

- Análisis de las justificaciones y comentarios a las preguntas. Los resultados se presentan en porcentaje, ya que es un análisis de frecuencia de aparición de los comentarios para cada ítem de la encuesta.

- Resultados de la encuesta. Los resultados son las calificaciones que cada encuestado dio a los ítem de la encuesta en una escala de 1 a 7.

- Comparación entre los resultados de la encuesta y los comentarios y justificaciones de los resultados.

- Verificación del cumplimiento de las hipótesis.

\subsection{Análisis de Justificaciones y Comentarios a las Preguntas}

Al momento de aplicar el cuestionario, se solicitó a los encuestados que justificaran y/o comentaran lo que les pareciera pertinente en cualquier momento de la entrevista. Por lo tanto, el $100 \%$ se refiere al total de personas que comentaron y/o justificaron cada pregunta, es decir, el $100 \%$ de una pregunta puede contener 50 comentarios y el $100 \%$ de otro pregunta puede contener 80 comentarios.

Los comentarios se agruparon de acuerdo a la caracterización presentada en la sección 3.2.2 (Profesionales versus técnicos y Personal de oficina central versus personal de obras). A continuación se presentan las diferencias más importantes encontradas.

\subsubsection{Profesionales versus técnicos}

- Enfoque al Cliente

En cuanto al conocimiento de los requerimientos del mandante, las opiniones del personal Técnico se dividen entre la apreciación de que "se transmiten bien hasta el nivel de profesionales" (43\%), que "existen esfuerzos de transmitirlos al resto de personal" (29\%) y que "Ios trabajadores deben conocer los requerimientos de la empresa" (29\%). En resumen, la gran mayoría coincide en que es importante que todo el personal conozca los requerimientos del mandante para que resulte una vivienda de buena calidad.

Por otra parte, las opiniones del personal Profesional se dividen entre los que creen que "todo el personal debe conocer los requerimientos del mandante" $(26 \%)$, los que creen que "hay una buena difusión de estos requerimientos hasta el nivel de profesionales" $(37 \%)$, los que opinan que "no es necesario que los trabajadores (técnicos y mano de obra no calificada) conozcan los requerimientos del mandante" (5\%) y los que creen que "no están capacitados para entenderlos y por eso no se les dan a conocer" (16\%). En resumen, no todo el personal a cargo de difundir estos conceptos está convencido de que sea lo más correcto o más útil y por lo tanto, esta apreciación puede ser la causa de que no todos los trabajadores conozcan los requerimientos del mandante.

Respecto a la satisfacción del cliente, aproximadamente un tercio de los profesionales opinan que "sus clientes están satisfechos con las viviendas entregadas" y los otros dos tercios de los profesionales piensan que "el cliente no sabe qué exigir y por lo tanto hace aumentar en demasía el trabajo de post-venta". Los Técnicos coinciden en que el cliente no sabe qué exigir.

Relacionado con lo anterior está lo relacionado con el cliente interno. Las opiniones de los Profesionales se distribuyen casi uniformemente entre los que piensan, por un lado, que "Ios requisitos están poco claros", que "Ios requisitos son variables en el tiempo" y por otro lado, entre los que creen que "hay un mayor compromiso en la oficina central con respecto a la calidad de las viviendas" y los que creen que "el mayor compromiso está en las obras". El personal Técnico coincide en un $100 \%$ en que "los requisitos del cliente interno son distintos de los del cliente final" y no opinan sobre dónde está el mayor compromiso con la calidad de las viviendas. Respecto a la posible diferencia entre los requisitos del cliente final y el cliente interno, al parecer el problema 
radica en que los mandos medios y altos no han sabido transmitir y traducir los requerimientos del cliente final a todo el personal. Estos niveles se encargan de traducir los requerimientos del cliente final a un programa de trabajo y de costos. Posteriormente, entregan a los niveles jerárquicos más bajos ambos programas (entre otras cosas) y exigen su cumplimiento, pero no se preocupan de explicar las razones de cada uno y lo único que ven los niveles más bajos es que se les pide terminar sus tareas en un tiempo mínimo y para poder lograrlo, se sienten obligados a dejar la calidad de lado, por lo que entienden que lo que les piden se contrapone con lo requerido por el cliente final. De todos modos este comentario es sólo una opinión, ya que este análisis no es parte de esta investigación profundizar en este tema.

\section{- Participación del Personal}

Respecto a este punto sólo opinaron los Profesionales, de ellos, casi la mitad (44\%) piensa que "la Dirección de la empresa escucha sus sugerencias de mejoramiento pero que rara vez las aplican". Sin embargo, el 38\% piensa que "su empresa fomenta la participación de todos", lo que es contradictorio.

\section{- Enfoque Basado en Procesos}

Cuando se les pregunta a los encuestados si conocen los factores que influyen en la calidad de las viviendas, el $50 \%$ de los Profesionales y el $100 \%$ de los Técnicos responden que "no se pueden conocer todos los factores porque existen muchos imprevistos". A esto se le debe poner especial atención, ya que si se considera que existen tantos imprevistos, podría pensarse que los procesos son incontrolables y a la vez difíciles de manipular para obtener mejores resultados. Esto también muestra cierto grado de pesimismo entre las personas, que pueden llegar a pensar que no importa el esfuerzo ni cuál sea la herramienta que se intenta aplicar, porque podrían pensar que no se obtendrán los resultados esperados y por lo mismo no los aplicarán. Es importante detectarlo y tomar medidas al respecto, ya que sólo así se obtendrán mejoras.

\section{- Enfoque de Sistema para la Gestión}

Con respecto a la comunicación, sólo el 17\% de los Profesionales opinaron que "en su empresa existe una buena comunicación" y el 58\% piensa que "falta crear más canales de comunicación transversales", entendiendo por comunicación transversal el traspaso de información de una obra a otra para que los conocimientos queden dentro de la empresa.

Con este resultado queda claro que en el día a día en una construcción, la carrera por solucionar problema tras problema, ocupa la mayor parte del tiempo, quedando relegado a segundo plano el tema de la comunicación y la gestión del conocimiento.

A modo de opinión se puede decir que las obras de construcción deberían ser mejor planificadas de lo que Io son en la actualidad, no sólo respecto a los métodos y secuencias constructivas, sino que respecto también a la gestión de la información y del conocimiento. Las herramientas existen, entonces, las constructoras desde sus niveles más altos deben buscar las herramientas más adecuadas a su organización e implementarlas organizada y planificadamente y midiendo su efectividad. Por ejemplo, una herramienta simple de implementar y de utilizar es un repositorio de soluciones a problemas. En la intranet de la empresa se puede mantener este repositorio que debe ser llenado con las soluciones satisfactorias de cada una de las obras para problemas de distinto tipo, entonces, cuando en una obra surja un problema, se pueda consultar, probar la solución que funcionó en otra parte y/o complementarla con ideas propias.

\section{- Mejora Continua}

Respecto de las no conformidades, existen visiones distintas entre los Profesionales y Técnicos. Por un lado, los Profesionales creen que "las no conformidades se entienden como una forma de castigo" (aproximadamente 39\%), que "el personal desvirtúa las no conformidades usándolas para cubrir sus espaldas" (alrededor del $39 \%$ ) y sólo un $23 \%$ cree que se entienden como una forma de mejorar los procesos. Por otro lado, a nivel Técnico, el $60 \%$ piensa que "el personal las entiende como una forma de mejorar los procesos". Esto evidencia una visión mucho más positiva entre los Técnicos. Sin embargo, los encuestados siempre se refieren al resto, ellos siempre dicen entender las no conformidades como una instancia de crecimiento y mejora.

- Enfoque Basado en Hechos para la Toma de Decisión No todos los Profesionales están de acuerdo respecto de la medición de indicadores (forma de evidenciar el estado y el grado de cumplimiento de los procesos y productos): el $45 \%$ piensa que "se están midiendo los más importantes", sin embargo, el $40 \%$ cree que "se miden sólo por cumplir y en realidad no se están utilizando para retroalimentar los procesos". Esto podría significar que las empresas están generando información importante y no saben utilizarla o que realmente están midiendo parámetros sólo por cumplir con la norma ISO 9001:2000.

- Relaciones Mutuamente Beneficiosas con los proveedores (abastecedores de recursos y/o de servicios)

Antes de presentar el análisis cabe mencionar que, reconociendo que los abastecedores de recursos y 
el de servicios tienen características distintas, en esta investigación no es relevante, ya que se consulta por la relación entre la constructora y el proveedor, específicamente en temas relacionados con la comunicación entre ambos que pueden darse en uno y otro caso.

En cuanto a este principio de gestión de la calidad, se observan apreciaciones muy distintas entre Profesionales y Técnicos. Los Profesionales opinan que "las empresas buscan compartir las responsabilidades con los proveedores" (30\%), también "consideran importante compartir la información del proyecto con el proveedor" (26\%), pero también algunos piensan que "los problemas de calidad de las viviendas son por culpa de los proveedores" (30\%), Ilámese a estos abastecedores o subcontratistas. En cambio a nivel Técnico, una gran parte de los encuestados piensa que "los proveedores cumplen con sus responsabilidades" $(33 \%)$ y que el problema es que "cuando la oficina central se encarga de comprar los materiales, son ellos los que demoran la entrega" (33\%), lo que implica un atraso de las obras. Estas apreciaciones tan distintas hacen pensar en que probablemente las expectativas (o los requerimientos) sobre la calidad del trabajo de los proveedores son distintas en ambos grupos.

\subsubsection{O ficina Central versus O bras}

\section{- Enfoque al Cliente}

A nivel de 0 ficina Central, el $67 \%$ del personal entrevistado piensa que "conocer los requerimientos del cliente (quien comprará la vivienda para habitarla) es problema sólo de la inmobiliaria", por lo que ellos como constructora no deben preocuparse por este tema. En cambio, el personal de O bra piensa que "es muy importante para la constructora saber cuáles son los requerimientos del cliente" (67\%). Lo que refleja que en Oficina Central tienen claro lo concerniente al proyecto y al contrato con la inmobiliaria, pero en Obra al momento de construir surgen las dudas y se hace necesario saber qué es lo que realmente quiere el cliente para poder entregar una vivienda que cumpla con sus expectativas.

Ahora, respecto al conocimiento de los requerimientos del mandante (puede entenderse como inmobiliaria), ambos grupos coinciden en que "existe una buena difusión hasta el nivel de profesionales" (oficina central: 39\%; obra: $46 \%$ ), en que "los trabajadores no están capacitados para entenderlos" (oficina central: 7\%; obra: $7 \%$ ) y además "no les interesa conocerlos" (oficina central: 15\%; obra: $15 \%$ ), pero también coinciden en que "todos los trabajadores deberían conocer los requerimientos de la empresa constructora" (oficina central: 23\%; obra: $15 \%)$. Entonces queda claro que es necesario involucrar a todos los trabajadores y que primero se debe motivarlos a participar en el SGC.

Respecto a la satisfacción del cliente, sólo el $15 \%$ del personal de la oficina central de las constructoras cree que "sus clientes están satisfechos" y el 57\% piensa que "el cliente no sabe qué exigir", es decir, subestiman al cliente. Por otra parte, el $100 \%$ del personal entrevistado en obra que comentó sobre este tema, también cree que "el cliente no sabe qué exigir". Con esto queda claro que es necesario capacitar y motivar al personal respecto de la calidad de las viviendas, de sus procesos constructivos y del SGC.

Desde el punto de vista del cliente interno (si se entiende el proceso de producción como una cadena de procesos, cada proceso es cliente del anterior, es su cliente interno), el $67 \%$ del personal de obra piensa que "los requerimientos del cliente interno son distintos de los requerimientos del cliente final, se contraponen" y que "hay mucho más compromiso con la calidad de las viviendas en las obras que en la oficina central" (33\%). El personal de oficina central cree que "Ios requerimientos del cliente son poco claros" (29\%) e igual proporción piensa que "estos requisitos son variables en el tiempo". Claramente se necesita una capacitación respecto de los requerimientos del cliente interno y final, por lo menos una pequeña explicación para que no surja este tipo de discordancias.

\section{- Liderazgo}

Respecto a este tema, ambos grupos coinciden en sus apreciaciones y corroboran las especulaciones anteriores. Creen que "falta motivar al personal respecto de la calidad" (oficina central: $37 \%$; obra: $24 \%$ ) y que "la difusión de políticas y objetivos de calidad es más teórica que práctica" (oficina central: 23\%; obra: 24\%).

\section{- Enfoque Basado en Procesos}

Respecto del conocimiento de los factores que influyen en la calidad de las viviendas, el personal de obra es más drástico que el de oficina central. Ellos piensan que "existen muchos imprevistos en la construcción y por eso no se pueden conocer todos los factores que influyen en la calidad de las viviendas" (80\%). En cambio el personal de oficina central además de pensar que "existen muchos imprevistos" (44\%), también creen que "a nivel de obra se manejan bien estos factores" (11\%) y que "falta que el personal conozca mejor su participación en cada proceso" $(22 \%)$.

- Enfoque de Sistema para la Gestión

Sobre la disponibilidad de recursos las opiniones 
están divididas. Mientras el personal de oficina central piensa que "no siempre están disponibles en forma oportuna porque existen problemas de flujo de caja en la empresa" (27\%), "por problemas de burocracia interna" (36\%), o "por problemas de planificación" (9\%), otra importante proporción cree que "no hay problemas de disponibilidad de recursos" (27\%). Por otra parte, el personal de obra piensa que "Ios recursos no están disponibles en forma oportuna por diferencias de objetivos entre la oficina central y las obras" $(46 \%)$, "por problemas de flujo de caja" (18\%), "problemas del proveedor" (18\%), "mala planificación" (9\%) o "por burocracia" (9\%). Por lo visto, el principal problema es de comunicación, ya que no puede ser que los grandes objetivos sean distintos ni que a nivel central piensen que no hay problemas con recursos siendo que en las obras si existen. Reforzando la teoría anterior, el 50\% del personal de oficina central piensa que "faltan canales de comunicación transversales" mientras el $100 \%$ del personal de las obras piensa lo mismo.

\section{- M ejora Continua}

Respecto al uso de las no conformidades, en obra el $67 \%$ piensa que "el resto las entienden como castigo", dejando claro que cada entrevistado dice no verlas de ese modo. En oficina central sólo el $30 \%$ piensa lo mismo. Sin embargo, en oficina central el $40 \%$ cree que "se usan para cubrir espaldas". Con estos resultados se hace evidente que la mayor parte del personal no sabe, no entiende o no quiere usar esta herramienta de mejoramiento. Habría que estudiar si es por falta de capacitación o de motivación. En cuanto a la prevención de las no conformidades, la mayor parte de ambos grupos coincide en que "se previenen constantemente" (oficina central: $50 \%$; obra: $42 \%$ ).

- Enfoque Basado en Hechos para la Toma de Decisión Respecto a la medición de indicadores existe una discrepancia. Por un lado, el $67 \%$ del personal de obra dice que "se están midiendo los parámetros más importantes" y el $11 \%$ piensa que "no se están midiendo los más importantes". Por otro lado, sólo el 33\% del personal de oficina central cree que "se miden los indicadores más importantes" y el $42 \%$ piensa que "no se miden los más importantes". Puede ser que lo que un grupo considera importante, para el otro grupo no lo sea tanto. En resumen, al analizar sólo los comentarios y justificaciones del personal de oficina central y de obras, parece haber cierta discrepancia en varios temas entre estos dos grupos. Además, al parecer no todo el personal está convencido de la utilidad y eficacia del SGC. Más adelante se volverán a analizar estos argumentos junto a los resultados de las encuestas.

\subsection{Resultados de la encuesta}

Estos resultados se presentarán en dos etapas: 1) análisis de las medias (promedios) y, 2) análisis de la varianza de los resultados de acuerdo a la caracterización hecha en la sección 3.2.2.

\section{Análisis de M edias}

Se calcularon algunos estadígrafos para cada dimensión (ver tabla 3). Cabe destacar que estos cálculos se realizaron luego de efectuadas las transformaciones pertinentes para las preguntas formuladas de manera inversa (preguntas en las cuales un puntaje alto significa un incumplimiento de alguna variable) y de ponderar las preguntas de acuerdo a los resultados de los análisis factoriales.

Las puntuaciones moderadas obtenidas, significan que los trabajadores de cada empresa no consideran óptimo el cumplimiento de los principios de gestión de la calidad, por lo que habría que ver cuál es el motivo: o no es posible hacerlo con los recursos disponibles o no lo consideran útil.

Tabla 3. Información estadística de las dimensiones

\begin{tabular}{||l|c|c|c|c||}
\hline \multicolumn{1}{||c|}{ Dimensión } & Media & Mínimo & Máximo & $\begin{array}{c}\text { Desviación } \\
\text { estándar }\end{array}$ \\
\hline \multicolumn{1}{||l|}{\begin{tabular}{l} 
Principios de Gestión de la Calidad \\
\hline Enfoque al Cliente (relación indirecta)
\end{tabular}} & 5.9 & 1.6 & 7.0 & 0.93 \\
\hline Enfoque al Cliente (relación directa) & 5.6 & 1.5 & 7.0 & 1.10 \\
\hline Liderazgo & 5.9 & 3.2 & 7.0 & 0.82 \\
\hline Participación del Personal & 6.3 & 4.0 & 7.0 & 0.71 \\
\hline Enfoque Basado en Procesos & 6.1 & 4.0 & 7.0 & 0.75 \\
\hline Enfoque de Sistema para la Gestión & 5.8 & 2.7 & 7.0 & 0.92 \\
\hline Mejora Continua (difusión de prácticas) & 5.9 & 3.6 & 7.0 & 0.84 \\
\hline Mejora Continua (uso de herramientas) & 5.5 & 1.0 & 7.0 & 1.49 \\
\hline Enfoque Basado en Hechos para la toma de decisión & 6.0 & 3.0 & 7.0 & 0.98 \\
\hline $\begin{array}{l}\text { Relación mutuamente Beneficiosa con el Proveedor (relación } \\
\text { contractual) }\end{array}$ & 5.3 & 1.8 & 7.0 & 1.03 \\
\hline $\begin{array}{l}\text { Relación mutuamente Beneficiosa con el Proveedor (relación } \\
\text { extra- contractual) }\end{array}$ & 5.7 & 1.9 & 7.0 & 0.87 \\
\hline
\end{tabular}


El promedio más alto es de la "participación del personal" (6.3) y el promedio más bajo es de las "relaciones mutuamente beneficiosas con el proveedor", la parte correspondiente a la relación contractual (5.3). El promedio más alto muestra que el personal realmente cree que es escuchado por la dirección de la empresa. Por otro lado, sólo otorga una calificación suficiente a la relación contractual con el proveedor, que apunta principalmente a la comunicación y traspaso de información importante para el buen desarrollo de las obras y al cumplimiento de los requerimientos por parte del proveedor. De esto se podría entender que los problemas de incumplimiento por parte del proveedor se deben parcialmente, al problema de comunicación existente entre ambas partes.

Las dimensiones "participación del personal" y "enfoque basado en procesos" tienen como puntuación mínima un 4.0, es decir, no presentan puntuaciones insuficientes. Esto muestra que las empresas cuentan con el punto de partida para obtener resultados eficientes: ven las actividades de producción como una cadena de procesos y escuchan las ideas del personal, ya que son ellos los que constantemente están en contacto con los procesos y por lo tanto, los que con mayor facilidad pueden detectar instancias de mejora.

Análisis de la varianza de acuerdo a la caracterización

Como forma de estudiar los resultados obtenidos desde distintos puntos de vista, se realizó un análisis de la varianza (ANOVA) para las categorías en que se dividió la muestra de acuerdo a la caracterización realizada en la sección 3.2.2. El objetivo es examinar las diferencias entre los distintos grupos, para verificar su significación estadística. Es decir, se estudió la diferencia de promedios (medias) del cumplimiento de los principios de gestión de la calidad.

Se estudiaron las diferencias entre los grupos de acuerdo a la caracterización realizada anteriormente porque un SGC apunta a mejorar continuamente y para esto, todas las personas implicadas deben tener la misma visión de la situación actual de cada empresa y deben tener las mismas metas y/o ser éstas complementarias. Entonces, al analizar la varianza de los distintos grupos se puede detectar si hay concordancia entre ellos o no respecto a estos temas.

Clasificación de acuerdo al Lugar de Trabajo Al realizar el análisis general se detectó que no existen diferencias significativas estadísticamente entre las medias de los grupos de personal de O ficina Central y personal de 0 bra. Los resultados se presentan en la tabla 4.

Tabla 4. Estadígrafos para analizar la diferencia entre personal de $\mathrm{O}$ ficina Central y de $\mathrm{O}$ bra en general

\begin{tabular}{||c|c|c|c|c||}
\hline \hline & $\begin{array}{c}\text { Media } \\
\text { Oficina } \\
\text { Central }\end{array}$ & $\begin{array}{c}\text { Media } \\
\text { Obra }\end{array}$ & F & Sig. \\
\hline PGC & 5.71 & 5.79 & 0.434 & 0.511 \\
\hline
\end{tabular}

Al realizar un análisis más detallado de las diferencias entre estos grupos, se observan diferencias importantes entre las apreciaciones del personal de oficina central y de obra respecto al liderazgo y el enfoque basado en procesos, diferencias leves en las dimensiones de mejora continua, relaciones mutuamente beneficiosas con el proveedor, participación del personal, enfoque al cliente, relación indirecta y no se observan diferencias significativas en las dimensiones enfoque al cliente, relación directa, enfoque de sistema para la gestión y enfoque basado en hechos para la toma de decisión, como se observa en la tabla 5 . Estos resultados de analizarán en conjunto con los resultados de los comentarios y justificaciones en la sección siguiente.

Tabla 5. Estadígrafos para analizar la diferencia entre 0 ficina Central y $\mathrm{O}$ bra en detalle por dimensiones

\begin{tabular}{|l|c|c|c|c||}
\hline & $\begin{array}{c}\text { Media } \\
\text { Oficina } \\
\text { Central }\end{array}$ & $\begin{array}{c}\text { Media } \\
\text { Obra }\end{array}$ & F & Sig. \\
\hline Enfoque al Cliente (relación indirecta) & 5.21 & 5.21 & 0.000 & 0.992 \\
\hline Enfoque al Cliente (relación directa) & 5.00 & 4.59 & 2.568 & 0.112 \\
\hline Liderazgo & 5.28 & 5.83 & 11.378 & 0.001 \\
\hline Participación del Personal & 6.05 & 6.26 & 1.570 & 0.213 \\
\hline Enfoque Basado en Procesos & 5.86 & 6.23 & 5.818 & 0.017 \\
\hline Enfoque de Sistema para la Gestión & 5.72 & 5.72 & 0.000 & 0.988 \\
\hline Mejora Continua (difusión de prácticas) & 5.59 & 5.95 & 2.981 & 0.087 \\
\hline Mejora Continua (uso de herramientas) & 5.46 & 5.08 & 0.968 & 0.328 \\
\hline $\begin{array}{l}\text { Enfoque Basado en Hechos para la toma de } \\
\text { decisión }\end{array}$ & 5.85 & 5.84 & 0.002 & 0.963 \\
\hline $\begin{array}{l}\text { Relación mutuamente Beneficiosa con el } \\
\text { Proveedor (relación contractual) }\end{array}$ & 4.99 & 5.30 & 1.332 & 0.251 \\
\hline $\begin{array}{l}\text { Relación mutuamente Beneficiosa con el } \\
\text { Proveedor (relación extra- contractual) }\end{array}$ & 5.50 & 5.04 & 1.467 & 0.229 \\
\hline \hline
\end{tabular}


Clasificación de acuerdo al Nivel Educacional

Al realizar el análisis general no se detectaron diferencias significativas estadísticamente. Los resultados se presentan en la tabla 6.

Tabla 6. Estadígrafos para analizar la diferencia entre personal Profesional y Técnico en general

\begin{tabular}{||c|c|c|c|c||}
\hline & $\begin{array}{c}\text { Media } \\
\text { Profesionales }\end{array}$ & $\begin{array}{c}\text { Media } \\
\text { Técnicos }\end{array}$ & F & Sig. \\
\hline PGC & 5.74 & 5.80 & 0.270 & 0.604 \\
\hline
\end{tabular}

Al realizar un análisis más detallado, se observan diferencias importantes entre las apreciaciones del personal profesional y técnico en las dimensiones enfoque basado en procesos, liderazgo y relaciones mutuamente beneficiosas con el proveedor, relación extra-contractual y diferencias leves en las otras dimensiones. Los resultados se muestran en la tabla 7.

Tabla 7. Estadígrafos para analizar la diferencia entre Profesionales y Técnicos en detalle por dimensiones

\begin{tabular}{||l|c|c|c|c||}
\hline & $\begin{array}{c}\text { Media } \\
\text { Profesionales }\end{array}$ & $\begin{array}{c}\text { Media } \\
\text { Técnicos }\end{array}$ & F & Sig. \\
\hline Enfoque al Cliente (relación indirecta) & 5.25 & 5.16 & 0.293 & 0.589 \\
\hline Enfoque al Cliente (relación directa) & 4.66 & 4.76 & 0.177 & 0.675 \\
\hline Liderazgo & 5.55 & 5.85 & 3.854 & 0.052 \\
\hline Participación del Personal & 6.17 & 6.24 & 0.260 & 0.611 \\
\hline Enfoque Basado en Procesos & 5.99 & 6.30 & 5.106 & 0.026 \\
\hline Enfoque de Sistema para la Gestión & 5.76 & 5.66 & 0.275 & 0.601 \\
\hline Mejora Continua (difusión de prácticas) & 5.78 & 5.93 & 0.613 & 0.435 \\
\hline Mejora Continua (uso de herramientas) & 5.24 & 5.13 & 0.094 & 0.760 \\
\hline $\begin{array}{l}\text { Enfoque Basado en Hechos para la } \\
\text { toma de decisión }\end{array}$ & 5.80 & 5.91 & 0.216 & 0.643 \\
\hline $\begin{array}{l}\text { Relación mutuamente Beneficiosa con } \\
\text { el Proveedor (relación contractual) }\end{array}$ & 5.11 & 5.36 & 1.065 & 0.304 \\
\hline $\begin{array}{l}\text { Relación mutuamente Beneficiosa con el } \\
\text { Proveedor (relación extra- contractual) }\end{array}$ & 5.39 & 4.88 & 2.254 & 0.137 \\
\hline \hline
\end{tabular}

De lo anterior se puede entender que las realidades del personal de oficina central y del personal de obra son más distintas entre sí que las realidades del personal profesional y técnico. Esto puede deberse a que ninguno de los grupos conoce las dificultades ni los progresos del otro grupo y por lo tanto tienen visiones distintas, en particular, del desempeño del SGC. En cambio, en ambos lugares profesionales y técnicos trabajan juntos, por lo tanto enfrentan los mismos problemas y encuentran sus soluciones. Las discrepancias entre profesionales y técnicos puede deberse a que su capacidad de decisión es distinta y a que el grupo técnico normalmente se encuentra sometido a las decisiones de los profesionales y a la capacidad que tengan estos últimos de difundir nuevas prácticas, en especial, sobre el SGC.
4.3 Comparación entre los resultados de la encuesta y los comentarios y justificaciones de las respuestas

En la sección anterior se mostraron los resultados obtenidos de las encuestas y las diferencias entre los distintos grupos. En esta sección se buscó si las diferencias detectadas en los resultados entre los distintos grupos, también se reflejan en los comentarios de los encuestados.

- Enfoque al Cliente

Respecto a este principio, los resultados de la encuesta difieren de los comentarios y justificaciones. Los resultados de la encuesta no presentan diferencias importantes entre las percepciones de los distintos grupos, especialmente entre el personal de oficina central y el de obras, pero de acuerdo a los comentarios emitidos por los encuestados, existen diferencias en cuanto a que la mayoría del personal de las obras cree que a la constructora le interesa conocer los requerimientos del cliente final a todo nivel y el personal de oficina central piensa que sólo le interesa a la inmobiliaria, porque quienes habitarán las viviendas que construye no son su cliente directo.

\section{- Liderazgo}

Las justificaciones y comentarios de los encuestados, también difieren de los resultados de la encuesta en lo relacionado con este principio. Los resultados del análisis entre oficina central y las obras arroja diferencias importantes entre estos grupos, en cambio, los comentarios y justificaciones sólo muestran pequeñas variaciones en las percepciones de cada uno, excepto en la apreciación de la difusión a todo nivel de los objetivos de la calidad, donde aproximadamente el doble de personal de obra que de oficina central piensa que esta difusión a todo nivel es real $(17 \%$ de obra, contra $7 \%$ de oficina Central).

- Enfoque Basado en Hechos para la Toma de Decisión En cuanto a los resultados de la encuesta no se aprecian diferencias, pero de acuerdo a los comentarios existe una gran diferencia respecto a qué indicadores son importantes para cada grupo, ya que el personal de oficina central y el personal profesional cree que no se están midiendo los más importantes y que además, no se están utilizando adecuadamente los que se miden y por otro lado, el personal de obra y el personal técnico creen que si.

Respecto a las otras dimensiones, no se observan discrepancias destacables entre los resultados de la 
encuesta y los comentarios de los encuestados por lo que no se ahondará en ellos.

\section{Verificación del cumplimiento de la hipótesis}

La hipótesis planteada fue la siguiente: "Ias empresas que tienen un sistema de gestión de la calidad certificado, cumplen apropiadamente los principios de gestión de la calidad".

Para evaluar la hipótesis, se realizaron dos análisis: 1) se estudiaron las medias de los principios de gestión de la calidad para determinar si los encuestados perciben un mejoramiento en el cumplimiento de los principios de gestión de la calidad y, 2) se analizó la varianza entre los grupos definidos en la caracterización de la muestra (sección 3.2.2) para determinar si existen diferencias significativas estadísticamente entre estos grupos. Esto último se presentó en la sección 4.2.

\subsection{Análisis de M edias}

Como se puede observar en la tabla 8 , todos los principios de gestión de la calidad fueron calificados con notas suficientes como mínimo por todos los grupos (calificación mínima: 4,59). Esto significa que consideran que se ha aumentado el cumplimiento de estos principios desde que se implementó el SGC en mayor o menor grado.

El principio que obtuvo la más alta calificación en casi todos los grupos es la participación del personal.
Esto muestra que se sienten escuchados por la dirección y que se sienten partícipes del SGC.

El principio con la segunda calificación más alta es el enfoque basado en procesos, lo que muestra que las empresas han confiado en este enfoque y han adoptado esta visión para administrar su producción.

El enfoque al cliente se ubica dentro de los principios peor calificados, siendo que lo fundamental de un SGC es orientar los esfuerzos a la satisfacción del cliente. Esto no quiere decir que a la dirección de la empresa no le interese satisfacerlos, sino que el personal percibe que de todos los principios es uno de los que menos atención le da la dirección, de acuerdo a lo que les es transmitido por sus superiores. Por lo tanto, las empresas deberían poner atención en la motivación de los mandos medios para transmitir a sus subalternos los principios de gestión de la calidad y en la capacitación que se realiza sobre este tema a todo el personal.

Un punto positivo que se extrae de los resultados presentados anteriormente, es que en uno de los primeros lugares (tercero o cuarto, dependiendo del grupo) está el enfoque basado en hechos para la toma de decisión, el que fue calificado entre 5.8 y 5.9. Esto significa que las empresas tienen la intención de mejorar "organizadamente", es decir, están buscando parámetros de desempeño de sus procesos y productos (viviendas), los están midiendo y tienen la intención de utilizarlos para mejorar. Sin embargo, los comentarios indican que a pesar de la calificación, el personal está dividido entre los que creen que se están midiendo los indicadores más importantes y los que creen lo contrario.

Tabla 8. Estadígrafos para evaluar la hipótesis

\begin{tabular}{||l|c|c|c|c||}
\hline & $\begin{array}{c}\text { Media } \\
\text { Of. } \\
\text { Central }\end{array}$ & $\begin{array}{c}\text { Media } \\
\text { Obra }\end{array}$ & $\begin{array}{c}\text { Media } \\
\text { Profesionales }\end{array}$ & $\begin{array}{c}\text { Media } \\
\text { Técnicos }\end{array}$ \\
\hline Enfoque al Cliente (relación indirecta) & 5.21 & 5.21 & 5.25 & 5.16 \\
\hline Enfoque al Cliente (relación directa) & 5.00 & 4.59 & 4.66 & 4.76 \\
\hline Liderazgo & 5.28 & 5.83 & 5.55 & 5.85 \\
\hline Participación del Personal & 6.05 & 6.26 & 6.17 & 6.24 \\
\hline Enfoque Basado en Procesos & 5.86 & 6.23 & 5.99 & 6.30 \\
\hline Enfoque de Sistema para la Gestión & 5.72 & 5.72 & 5.76 & 5.66 \\
\hline Mejora Continua (difusión de prácticas) & 5.59 & 5.95 & 5.78 & 5.93 \\
\hline Mejora Continua (uso de herramientas) & 5.46 & 5.08 & 5.24 & 5.13 \\
\hline $\begin{array}{l}\text { Enfoque Basado en Hechos para la toma de } \\
\text { decisión }\end{array}$ & 5.85 & 5.84 & 5.80 & 5.91 \\
\hline $\begin{array}{l}\text { Relación mutuamente Beneficiosa con el } \\
\text { Proveedor (relación contractual) }\end{array}$ & 4.99 & 5.30 & 5.11 & 5.36 \\
\hline $\begin{array}{l}\text { Relación mutuamente Beneficiosa con el } \\
\text { Proveedor (relación extra- contractual) }\end{array}$ & 5.50 & 5.04 & 5.39 & 4.88 \\
\hline
\end{tabular}




\subsection{Análisis de Varianza}

De acuerdo a lo presentado en la sección 4.2, existen mayores diferencias entre las apreciaciones de las personas de $\mathrm{O}$ ficina Central y de $\mathrm{O}$ bra que entre los Profesionales y los Técnicos. Entre la O ficina Central y las $\mathrm{O}$ bras, las diferencias más importantes se encuentran en los principios de gestión de la calidad "Liderazgo" y "Enfoque Basado en Procesos". Entre Profesionales y Técnicos, las diferencias más importantes se encuentran en estos dos principios y además, en el principio "Relaciones M utuamente Beneficiosas con el Proveedor, Relación Extra-Contractual".

Dejando a un lado las diferencias entre las apreciaciones de los distintos grupos, todos coinciden en que el cumplimiento de los principios de gestión de la calidad ha aumentado luego de implementar el SGC, de acuerdo a las calificaciones obtenidas por cada principio. Por otro lado, el análisis de la varianza arroja diferencias entre los distintos grupos, pero estas diferencias son matices que dependen de las expectativas de cada uno.

En resumen, los resultados apoyan la hipótesis, es decir, se ha aumentado el cumplimiento de los principios de gestión de la calidad, pero considerando que las calificaciones de cada principio no fueron tan altas, los comentarios y justificaciones negativos respecto al cumplimiento de algunos principios y a las discrepancias entre los grupos, este aumento del cumplimiento no ha sido en la medida que los encuestados desearían.

\section{Conclusiones, recomendaciones y perspectivas futuras}

Los resultados de esta investigación permiten establecer que las empresas constructoras habitacionales cumplen parcialmente con los principios de gestión de la calidad propuestos por la norma ISO 9001:2000, de acuerdo a las percepciones de los encuestados sobre este tema que, si bien son positivas, consideran sólo medianamente bueno el cumplimiento de los principios de gestión de la calidad.

Por otro lado, de acuerdo a los comentarios hechos por los encuestados, queda de manifiesto que la mayoría no está convencida de que la aplicación de esta norma les sea útil, más bien la ven como una fuente de trabajo extra. Además, no todos piensan que la aplicación del SGC haya propiciado las mejoras observadas, más bien creen que es lo que naturalmente ocurre cuando el personal hace bien su trabajo.

O tras conclusiones que evidencian el mediano cumplimiento de los principios de gestión de la calidad son las siguientes:

- Parte del personal piensa que es importante que todos conozcan los requerimientos del mandante, en cambio el personal a cargo de difundir estos conceptos no está convencido de que sea lo más correcto o lo más útil, lo que puede ser la causa de que no todos los trabajadores conozcan los requerimientos del mandante.

- Gran parte de los encuestados opinaron que sus clientes no saben qué exigir y por lo tanto hacen aumentar en demasía el trabajo de post-venta. Al parecer subestiman al cliente y no entienden o no se esfuerzan por entender sus requerimientos.

- Respecto al uso de las no conformidades (evidencia de una falla), los encuestados opinan que en general, el personal las entiende como una forma de castigo y que las desvirtúan utilizándolas para cubrirse las espaldas, pero destacan que ellos las ven como una instancia de crecimiento y mejora.

- Se detectó que las empresas podrían estar generando información importante a través de la medición de indicadores de desempeño y no saben utilizarla o realmente están midiendo parámetros sólo por cumplir con la norma ISO 9001:2000 y no los creen necesarios o no confían en ellos. De esto se desprende que los encuestados opinan que se están midiendo ciertos parámetros pero no se están utilizando para retroalimentar los procesos.

- Respecto al incumplimiento de los requerimientos por parte de los proveedores, se concluyó que se debe parcialmente a un problema de comunicación entre la constructora y el proveedor.

Cabe destacar que la mayoría de los encuestados coincide en que la aplicación de un SGC les ha ayudado a organizarse, a ordenar la gestión de los procesos, pero también coinciden en que les ha traído una mayor carga de trabajo al tener que completar y usar una mayor número de documentos (registros de calidad, evidencia de que se han seguido todos los pasos y se han revisado). Además, creen que es casi imposible cumplir en un 100\% la programación debido a un sinnúmero de imprevistos, es decir, no sienten que sea manejable por ellos cumplir o no con el programa de trabajo.

Respecto a la implementación de la norma ISO 9001:2000, muchas empresas piensan que al seguir paso a paso las indicaciones de esta norma lograrán mejorar la calidad como una receta de éxito, pero olvidan lo 
principal: un SGC requiere un cambio en la forma de enfocar la gestión de las empresas, requiere enfocarse en las necesidades del cliente (interno y externo) y con sólo seguir las indicaciones de la norma esto no se logra, es más, el enfoque al cliente es uno de los principios que el personal percibe con el más bajo cumplimiento. Lo anterior se debe, por un lado, a que el personal de las empresas está siguiendo las indicaciones de una norma que ellos perciben que no se adapta a sus necesidades y por otro lado, a que el personal de las empresas piensan que siguiendo estas indicaciones sin cambiar de raíz la forma de ver el sistema de producción van a mejorar. En el fondo, sus sistemas de gestión de la calidad funcionan en el papel perfectamente (la medida del desempeño de un sistema de gestión de la calidad es la mejora que ocurre en los sistemas de producción), pero en la práctica tienen varias falencias, por ejemplo, no todo el personal tiene las mismas metas finales, lo que se deduce de las diferencias de percepciones de calidad de cada uno (en lo que a calidad se refiere, lo que para algunos es muy bueno, para otros es inaceptable).

Aunque los resultados indican que el desempeño de los SGC no es tan eficiente ni tan bueno como se pretendía que fuera, los resultados también demuestran que existe una clara tendencia de las empresas a trabajar en base a indicadores, es decir, pretenden mejorar su gestión en base a conocimientos reales y no percepciones.

Como recomendación, para mejorar la aplicación de los principios de gestión de la calidad, las empresas deben volver a las raíces de la gestión de la calidad, deben entenderlas y a la vez entender su negocio (en este caso la construcción) para así encontrar su propia fórmula de mejoramiento. Todo esto fundamentado en el compromiso de la dirección con la gestión de la calidad. Además, las empresas deben potenciar el uso de los indicadores de desempeño para retroalimentar los procesos, ya que es una muy buena forma de fundar las políticas de mejora continua y de seguir un camino más directo hacia mejores estándares de calidad.

La presente investigación ha ayudado a determinar el grado de cumplimiento de los principios de gestión de la calidad en las constructoras habitacionales. Sin embargo, los resultados de la investigación dejan de manifiesto la necesidad de profundizar el estudio de algunos temas para lograr una comprensión más acabada. En primer lugar, falta complementar los resultados de esta investigación con un estudio de la satisfacción de los clientes internos y externos, ya que se debe determinar si la percepción de buena calidad que tienen las empresas constructoras (a nivel externo) y los trabajadores (a nivel interno) es igual a la que tienen sus respectivos clientes. Por otro lado, se propone incentivar a las empresas constructoras a establecer parámetros (indicadores) de acuerdo a los resultados de esta investigación para posteriormente evaluar el cumplimiento de los principios de gestión de la calidad a partir de datos (valores) más duros, no sólo percepciones subjetivas de personas ligadas a las empresas.

Finalmente, se propone estudiar la relación entre la capacitación del personal y el grado de implementación de un SGC, ya que esta investigación encontró dos puntos de vista contrarios: por un lado, la teoría dice que todo el personal debe estar capacitado y enterado de los objetivos y metodologías de gestión de los procesos y de la calidad y por otro lado, la percepción de quienes tienen el poder de decisión en las constructoras habitacionales dice que sólo es efectiva esta capacitación hasta el nivel de mandos medios.

\section{Referencias}

Bou Llusar J., Camison C. (2002), Development and Validation of a Perceived Business Q uality M easurement Instrument, Q uality Management Journal, 9, № 4, pp. $23-38$.

Cameron K., Sine W. (1999), A framework for organizational quality culture, Q uality Management Journal, 6, № 4, pp. 7 - 25.

Chan T., Q uazi H. (2002), O verview of quality management practices in selected Asian countries, Q uality M anagement Journal, 9, № 1, pp. 23 - 49.

Choon K., Wisner J. (2001), A Framework for Quality Improvement in the Transportation Industry, Q uality Management Journal, 8, № 1, pp. $9-22$.

Cook L., Verma R. (2002), Exploring the linkages between quality system, service quality, and performance excellence: service providers' perspectives, Q uality management Journal, 9, No 2, pp. $44-56$.

Detert, J., Jenni, R. (2000), An instrument for measuring quality practices in education, Quality Management Journal, 7, №3, pp. 20 - 37.

Dick G., Gallimore K., Brown J. (2001), Does ISO 9000 Give a Q uality Emphasis Advantage? A Comparison of Large Service and M anufacturing $O$ rganizations, Q uality Management Journal, 8, № 1, pp. 52 - 61 . Evans J. (1997), Critical linkages in the Baldrige Award Criteria: research models and educational challenges, Q uality Management Journal, 5, № 1, pp. 13 - 30.

Evans James R., Lindsay William (2000), Administración y Control de la Calidad, 4ta edición, International 
Thomson Editores, S.A. de C.V.

Figari C. (1996), Diagnóstico de la calidad en la construcción chilena, Tesis de Magíster, Departamento de Ingeniería y Gestión de la Construcción, Pontificia Universidad Católica de Chile.

Gideon H., Kusuma M . (?) Total quality management in construction industries: Problems and Progress, http://puslit.petra.ac.id/news/seminars/construct.htm.

González, C. (2001) Caracterización de la cultura organizacional de la construcción nacional y su influencia en estrategias de cambio, Tesis de Magíster, Departamento de Ingeniería y Gestión de la Construcción, Pontificia Universidad Católica de Chile.

Grandzol J., Gershon M. (1997), Which TQ M practices really matter: an empirical investigation, Quality Management Journal, 4, № 4, pp. 43 - 59 .

Gustafsson A., Ekdahl F., Johnson M. (2000), Linking customer satisfaction to product design: a key to success for Volvo, Q uality Management Journal, 7, № 1, pp. 27 $-38$.

Hacker M., N ewton T., Akinyele A. (2001), The impact of a performance management intervention on work processes and operational indicators, Quality Management Journal, 8, No 2, pp. 8 - 18. Jain B., Tabak F. (2002), O rganizational quality management in emerging economies, Q uality Management Journal, 9, № 2, pp. $10-24$.

Jayaram J., Handfield R., G hosh S. (1997), The application of quality tools in achieving quality attributes and strategies, Q uality Management Journal, 5, № 1, pp. 75 $-100$.

Keinath B., Gorski B. (1999), An empirical study of the Minnesota Q uality Award evaluation process, Q uality Management Journal, 6, № 1, pp. 29 - 39.

Kontoghiorghes C. (2003), Examining the association between quality and productivity performance in a service organization, Q uality M anagement Journal, 10 № 1, pp. $32-42$.

Lévy Mangin J. P., Varela Mallou J. (2003), Análisis Multivariable para las Ciencias Sociales, Prentice Hall, Pearson Educación S.A.

Mc Cracken M., Kaynak H. (1996), An empirical investigation of the relationship between quality and productivity, Q uality Management Journal, 3, №2, pp. $36-51$.

Michalisin M., White G. (2001), An Empirical Study of the Posturing-Implementation Gap in Q uality Management, Q uality M anagement Journal, 8, № 1, pp. 34 - 51.

Molina M. (1998), Estrategias para la implementación de la gestión de calidad en la construcción chilena considerando las características culturales del sector,
Tesis de Magíster, Departamento de Ingeniería y Gestión de la Construcción, Pontificia Universidad Católica de Chile. NAHB Research Center Staff (1997), The bottom line on quality, http://www.toolbase.org/tertiaryT.asp?TrackID =\&Categ ory $\mid \mathrm{D}=908 \&$ o ocumentID $=705$

O choa G . (1998), M etodología de diagnóstico de la calidad para empresas constructoras, Tesis de Magíster, Departamento de Ingeniería y Gestión de la Construcción, Pontificia Universidad Católica de Chile.

Reeves C., Bednar D., Lawrence R. (1995), Back to the beginning: what do customers care about in service firms? Q uality Management Journal, 3, № 1, pp. 56 72.

Roethlein C., Mangiameli P., Ebrahimpour M. (2002), Quality U.S. manufacturing industries: an empirical study, Q uality Management Journal, 9, №3, pp. 48 66.

Salegna G., Fazel F. (1995), An integrative framework for developing and evaluating a TQ M implementation plan, Quality Management Journal, 3, № 1, pp. $73-84$.

Salkind N eil J. (1999), Métodos de Investigación, Tercera Edición, Prentice Hall, México.

Serpell A., Labra M. (2003), Un sistema de evaluación de la calidad de la construcción de viviendas en Chile, Revista Ingeniería de Construcción, Vol. 18, №2, pp. $93-96$.

Smith G. (1998), Determining the cause of quality problems: lessons from diagnostic disciplines, Q uality Management Journal, 5, № 2, pp. 24 - 41.

Spencer B., Crosby L. (1997), Linking quality attributes with customer purchasing decisions: a comparison of two methods, Quality Management Journal, 5, № 1, pp. $35-45$.

Sun H. (2001), Comparing quality management practices in the manufacturing and service industries: learning opportunities, Q uality management Journal, 8, №2, pp. $53-71$.

Torbica Z., Stroh R. (2000), HO MBSAT - An instrument for measuring home buyer satisfaction, Q uality Management Journal, 7, № 4, pp. $32-44$.

Vass D., Kincade D. (1999), Relationship of TQM implementation and employee opinion survey: a study of three manufacturers, Q uality Management Journal, 6, № 1, pp. $60-73$.

Yoshida K. (1995), Revisiting Deming's 14 points in Light of Japanese business practices, Q uality M anagement Journal, 3, №1, pp. 14 - 30.

Yu C., Cochran D., Spencer B. (1998), Q uality management practices in China, Q uality Management Journal, 5, № 2, pp. $91-106$. 


\section{Anexo A: instrumento de medición}

\section{Cuestionario para entrevista}

El objetivo de este cuestionario es recopilar información de varias empresas y de distintos niveles dentro de su jerarquía, de manera de obtener una impresión sobre la implementación de sistemas de gestión de la calidad para evaluar su real impacto en la calidad de las viviendas y de los procesos.

La mayor parte de las preguntas están diseñadas de tal forma que se puedan responder en el formato de una escala tipo Likert con calificaciones valoradas de 1 a 7, donde:

1 es muy en desacuerdo/ muy malo, 4 es indiferente/ suficiente $y$,

7 es muy de acuerdo/ excelente.

\section{Preguntas}

\section{PREGUNTA}

\section{NOTA}

1. A la Empresa le interesa considerablemente conocer los requerimientos de quienes habitarán las viviendas

2. Los trabajadores conocen todos los requerimientos de los clientes de esta Obra

3. En general, los clientes están completamente satisfechos con las viviendas de esta Empresa

4. Conozco claramente a mi cliente interno

5. Conozco todos los requerimientos de mi cliente interno

6. Me interesa mucho satisfacer los requerimientos de mi cliente interno

7. La Política de Calidad de la Empresa orienta nuestros esfuerzos hacia un mismo objetivo

8. Los objetivos de la calidad para esta obra son bien conocidos por todos

9. Los objetivos de la calidad para esta obra son entendidos completamente por todos

10. Los objetivos de la calidad para esta obra son muy importantes para todo el personal

11. La Dirección nos motiva fuertemente a comprometernos con la calidad

12. La Dirección se esfuerza mucho en enseñarnos sobre cómo llegar a cumplir los objetivos de la calidad propuestos

13. Todo el personal se siente muy comprometido con la Empresa

14. La Dirección considera muy importantes las sugerencias que hacemos para mejorar las actividades en que participamos

15. Me siento muy reconocido cuando hago un buen trabajo

16. A la Empresa le interesa mucho capacitarnos para la calidad porque lo considera un beneficio para ambos

17. Contamos con todos los conocimientos necesarios para realizar perfectamente nuestro trabajo

\section{PREGUNTA}

18. Conocemos todos los factores que influyen en la calidad de la actividad (proceso) en que participamos

19. Conocemos completamente cómo influye la calidad de nuestras actividades (procesos) en las siguientes actividades

20. La Dirección considera muy importante que los recursos estén disponibles oportunamente

21. En general en las obras de esta Empresa, los recursos están disponibles en forma oportuna

22. La comunicación entre las distintas áreas de la Empresa que participan en la construcción de las viviendas es muy fluida

23. Todos comprendemos que nuestro trabajo influye mucho en el logro de los objetivos de calidad de la Empresa 


\section{Anexo B: ponderadores que \\ resultan del análisis factorial}

Tabla B-1. Ponderadores para pasar de los ítems a las variables del estudio

\begin{tabular}{|c|c|c|c|c|c|c|c|c|c|c|c|}
\hline & \multicolumn{11}{|c|}{ Prácticas de Gestión de la Calidad } \\
\hline & EC1 & EC2 & $\mathbf{L}$ & PP & EBP & ESG & MC1 & MC2 & EBH & RBP1 & RBP2 \\
\hline P 1 & -0.238 & 0.657 & & & & & & & & & \\
\hline P_2 & 0.164 & 0.181 & & & & & & & & & \\
\hline $\mathbf{P}^{-3}$ & & 0.343 & & & & & & & & & \\
\hline$P 4$ & 0.431 & -0.127 & & & & & & & & & \\
\hline P_5 & 0.452 & -0.163 & & & & & & & & & \\
\hline P_6 & 0.191 & 0.109 & & & & & & & & & \\
\hline P 8 & & & 0.185 & & & & & & & & \\
\hline P_9 & & & 0.193 & & & & & & & & \\
\hline $\mathbf{P} 10$ & & & 0.159 & & & & & & & & \\
\hline$P 11$ & & & 0.188 & & & & & & & & \\
\hline P_12 & & & 0.192 & & & & & & & & \\
\hline $\mathbf{P} 13$ & & & 0.082 & & & & & & & & \\
\hline P_14 & & & & 0.308 & & & & & & & \\
\hline P_16 & & & & 0.353 & & & & & & & \\
\hline P 23 & & & & 0.339 & & & & & & & \\
\hline P_18 & & & & & 0.500 & & & & & & \\
\hline P_19 & & & & & 0.500 & & & & & & \\
\hline P_20 & & & & & & 0.331 & & & & & \\
\hline P_21 & & & & & & 0.327 & & & & & \\
\hline P 22 & & & & & & 0.342 & & & & & \\
\hline P_24 & & & & & & & 0.298 & 0.367 & & & \\
\hline P_25 & & & & & & & 0.339 & & & & \\
\hline P_26 & & & & & & & & 1.340 & & & \\
\hline P_27 & & & & & & & 0.363 & -0.707 & & & \\
\hline P_32 & & & & & & & & & 0.336 & & \\
\hline P_33 & & & & & & & & & 0.333 & & \\
\hline P 34 & & & & & & & & & 0.331 & & \\
\hline P_35 & & & & & & & & & & 0.400 & -0.125 \\
\hline P 36 & & & & & & & & & & 0.335 & \\
\hline P_37 & & & & & & & & & & 0.418 & -0.229 \\
\hline P_38 & & & & & & & & & & -0.221 & 0.603 \\
\hline P_39 & & & & & & & & & & -0.169 & 0.532 \\
\hline$P 40$ & & & & & & & & & & & 0.220 \\
\hline P_41 & & & & & & & & & & 0.237 & \\
\hline
\end{tabular}

D onde:

EC1: Enfoque al cliente (relación indirecta). EC2: Enfoque al cliente (relación directa). L: Liderazgo.

PP: Participación del personal

EBP: Enfoque basado en procesos.

ESG: Enfoque de sistema para la gestión.
MC1: Mejora continua (difusión de prácticas). MC2: Mejora continua (uso de herramientas). EBH : Enfoque basado en hechos para la toma de decisión. RBP1: Relación mutuamente beneficiosa con el proveedor (relación contractual).

RBP2: Relación mutuamente beneficiosa con el proveedor (relación extra-contractual). 
Tabla B-2. Ponderadores para pasar de las variables a una calificación por encuestado

\begin{tabular}{|c|c|c|}
\hline & & PGC \\
\hline \multirow{11}{*}{$\begin{array}{l}\text { Principios de } \\
\text { Gestión de la } \\
\text { Calidad }\end{array}$} & EC1 & 0.1357 \\
\hline & EC2 & 0.0004 \\
\hline & $\mathbf{L}$ & 0.0200 \\
\hline & PP & 0.0976 \\
\hline & EBP & 0.0805 \\
\hline & ESG & 0.059 \\
\hline & MC1 & 0.0903 \\
\hline & MC2 & 0.0974 \\
\hline & EBH & 0.1605 \\
\hline & RBP1 & 0.0902 \\
\hline & RBP2 & 0.1697 \\
\hline
\end{tabular}

N ota: En ambas tablas se eliminaron los ponderadores menores que 0,1 en valor absoluto. 